\title{
PEPTIC ULCER
}

\section{Risk factors for ulcer development in patients with non-ulcer dyspepsia: a prospective two year follow up study of 209 patients}

\author{
P-I Hsu, K-H Lai, G-H Lo, H-H Tseng, C-C Lo, H-C Chen, W-L Tsai, H-S Jou, N-J Peng, \\ C-H Chien, J-L Chen, P-N Hsu
}

Gut 2002;51:15-20

Background and aims: A subset of non-ulcer dyspepsia (NUD) disorders can evolve into peptic ulcer disease. This prospective study attempted to determine the independent risk factors for ulcer formation in NUD patients, and compared the natural history of Helicobacter pylori positive and negative NUD subjects.

Methods: From May 1997 to April 1999, consecutive NUD patients were enrolled into the study. Endoscopy was performed routinely on enrolment, at the end of the second and 12th months, and whenever there was a dyspepsia attack. Patients were prospectively followed up for two years.

Results: Peptic ulcers occurred in 16 of 209 NUD patients during the two year follow up period.

See end of article for authors' affiliations

Correspondence to: Dr K-H Lai, Department of Internal Medicine, Kaohsiung Veterans General Hospital, 386 Ta-Chung 1 st Road, Kaohsiung, Taiwan, ROC; williamhsup@yahoo.com.tw

Accepted for publication 18 September 2001 Multivariate analysis of 13 host and bacterial factors demonstrated that advanced age lodds ratio 2.90), $\mathrm{H}$ pylori infection (odds ratio 3.59), and use of non-steroidal anti-inflammatory drugs (NSAID; odds ratio 4.46) were independently significant in predicting subsequent peptic ulcer development. NUD patients with all three risk factors had a 75\% (3/4) risk of developing peptic ulcer but the ulcer incidence in patients without any of the risk parameters was only $1.2 \%(1 / 84)$. The resolution rate of symptoms in the $H$ pylori positive NUD patients was similar to the $H$ pylori negative patients $157.9 \% \mathrm{v}$ $49.1 \%$; $95 \%$ confidence interval $(\mathrm{Cl})-5$ to 22$)$. However, rates for subsequent peptic ulcer and erosion development were significantly higher in $\mathrm{H}$ pylori positive patients than in $\mathrm{H}$ pylori negative patients (ulcer $12.6 \%$ v 3.5\%, 95\% Cl 1-16; erosion 23.2\% v $12.3 \%, 95 \% \mathrm{Cl} 1-21$ ).

Conclusion: A small but significant proportion of NUD patients develop peptic ulcer after long term follow up. H pylori infection, NSAID use, and advanced age are independent risk factors for subsequent ulcer formation. Follow up endoscopy is strongly indicated for an NUD patient with multiple risk factors for ulcer formation when symptoms recur.
$\mathrm{N}$ on-ulcer dyspepsia (NUD) is one of the most frequently encountered disorders in gastroenterology clinics worldwide. ${ }^{1-3}$ Previous studies showed that the cumulative risk of ulcer development in NUD patients ranged from $1 \%$ to $21 \%$ during a follow up period of $1-10$ years. ${ }^{4-7}$ It is important to point out that some patients eventually died of perforated peptic ulcers. ${ }^{7}$ These studies suggest that a subset of NUD evolves into peptic ulcer disease. However, the risk factors influencing the subsequent development of peptic ulcers in NUD patients remain unclear.

The clinical significance of Helicobacter pylori in upper gastrointestinal disorders has been confirmed recently. This infection plays an important role in the pathogenesis of acute and chronic gastritis, peptic ulcer disease, gastric adenocarcinoma, and mucosa associated tissue lymphoma. ${ }^{8}$ Although many researchers ${ }^{9-11}$ have investigated the relationship between NUD and $H$ pylori infection, the benefit of anti-H pylori therapy in NUD patients remains controversial. It is also interesting to note that although approximately half of NUD patients are not infected by $H$ pylori, ${ }^{9}$ the natural history of $H$ pylori negative NUD has not yet been studied. Therefore, large scale long term clinical investigations of patients with $H$ pylori negative NUD are urgently needed.

Hence in this prospective study we attempted to determine the risk factors for ulcer formation in patients with NUD and to investigate the long term clinical outcome of $H$ pylori positive and negative NUD patients.

\section{PATIENTS AND METHODS}

\section{Selection of patients}

Eligible participants in the study were those who sought medical care for dyspeptic symptoms (pain or discomfort cen- tred in the upper abdomen) that had been present for at least one month, provided their symptom scores were $\geqslant 3$ (see questionnaire below), and they had normal endoscopic findings or images of gastritis only (that is, no oesophageal, gastric, or duodenal erosions or ulcers, reflux oesophagitis, or malignancy). Exclusion criteria were: (1) previous history of peptic ulcer, (2) use of non-steroidal anti-inflammatory drugs (NSAID) within one month of endoscopy, (3) antibiotic ingestion within one month of endoscopy, (4) serious medical illness, (5) pregnancy, (6) previous history of anti- $H$ pylori therapy, (7) associated pancreaticobiliary tract diseases, (8) associated gastrointestinal malignancy, and (9) associated irritable bowel syndrome. To confirm that symptoms were solely dyspeptic in origin, pancreaticobiliary tract disease was excluded by sonography. Patients with predominant symptoms such as heartburn or acid regurgitation were not included, even if they had a normal oesophageal mucosa on endoscopy, to avoid the influence of gastro-oesophageal reflux disease. Irritable bowel syndrome was diagnosed on the basis of the presence of at least three of Manning's criteria. ${ }^{12}$

The diagnosis of $H$ pylori infection was based on at least two positive results of histological findings, the rapid urease test and the ${ }^{13} \mathrm{C}$ urea breath test. According to the original study design, $H$ pylori positive NUD patients were randomly assigned to either the anti-H pylori or control group. Patients in the
Abbreviations: NUD, non-ulcer dyspepsia; NSAID, non-steroidal anti-inflammatory drugs. 
anti-H pylori group were excluded from this study and their data are reported elsewhere. ${ }^{13}$

\section{Study design}

Once informed consent was obtained, patients received lansoprazole $30 \mathrm{mg}$ twice daily for one week, and were then prospectively followed up for two years. During follow up, subjects were allowed to take an intermittent short course (less than two weeks) of antacids or prokinetic agents. However, $\mathrm{H}_{2}$ receptor antagonists or proton pump inhibitors were not allowed except when peptic ulcer developed.

Endoscopy with biopsies for the rapid urease test and histological examination was performed on enrolment, at the end of the second and 12th months, and when severe dyspepsia occurred. Peptic ulcer was defined as a circumscribed mucosal break $5 \mathrm{~mm}$ or more in diameter, with a well defined ulcer crater, whereas smaller lesions $(<5 \mathrm{~mm}$ in diameter $)$ were classified as erosions. The extent of ulceration was measured by opening a pair of biopsy forceps of known span in front of the ulcer. Patients also undertook a urea breath test on enrolment, and at the end of second, 12th, and 24th months. Additionally, symptom scores were assessed on enrolment and at the end of the second, sixth, 12th, 18th, and 24th months and whenever dyspepsia recurred. Subjects and interviewers were blinded to $H$ pylori status. The study was approved by the medical ethics committee of the KaohsiungVeterans General Hospital, and all patients gave written consent.

To assess the significance of these patient characteristics, the following data were recorded for each patient: age, sex, blood type, smoking, alcohol, coffee and tea consumption, and history of dyspeptic symptoms. Prior to endoscopy, venous blood was obtained for estimation of serum gastrin 17 and pepsinogen I concentrations by radioimmunoassay.

\section{Endoscopy, biopsy, and urea breath test}

During endoscopy, biopsies over the antrum and body were obtained for the rapid urease test (with one specimen from the antrum and another from the body) and histological examination (two specimens from the antrum and another two from the body). The rapid urease test was performed according to our previous studies. ${ }^{14}{ }^{15}$ Specimens for histological examinations were fixed in $10 \%$ buffered formalin, embedded in paraffin, and sectioned. The sections were stained with a haematoxylin and eosin stain and a modified Giemsa stain to observe the presence of curved rod shape bacteria on the mucosal surface. ${ }^{16}$ Biopsy specimens were assessed by a histopathologist (H-HT), blinded as to the endoscopic features, and the data from the rapid urease and urea breath tests. Patients also undertook a urea breath test according to a standard validated European protocol. ${ }^{17}$

\section{Symptom questionnaire and patient subgroups}

A previously validated Tucci's questionnaire ${ }^{18}$ was administered to each patient. The questionnaire assessed four well categorised dyspeptic symptoms: epigastric pain or burning, postprandial fullness, nausea, and vomiting. Each symptom was graded $0-3$ according to its influence on the patient's activities; 0, absent; 1, mild (not influencing the patient's activities); 2, relevant (diverting from, but not causing modifications to usual activities); and 3, severe (interfering with the ordinary activities of the patient). The pattern of symptoms was subdivided according to Tosetti's classification ${ }^{19}$ : (1) "prevalent pain subgroup" defined as epigastric pain $\geqslant 2$ with any other symptoms $\leqslant 1 ;(2)$ "prevalent discomfort subgroup" defined as postprandial fullness and/or nausea and/or vomiting $\geqslant 2$, with epigastric pain $\leqslant 1$; and ( 3 ) "unclassifiable subgroup" defined as symptoms which do not fulfill the criteria for prevalent pain or prevalent discomfort subgroup.

During each follow up, a detailed history of NSAID ingestion was obtained. Patients were regarded as NSAID users if they had consumed NSAID for more than three days within one month before interview. History of NSAID use was corroborated where possible from the patient's notes, pharmacy review, and the referring doctor's letter.

\section{Radioimmunoassay}

Serum gastrin 17 was measured using commercial kits from Incstar Corporation, USA. Normal values ranged from 25 to 89 $\mathrm{pg} / \mathrm{ml}$. Concentrations of pepsinogen I were determined using a radioimmunoassay (Sorin Biomedica, Saluggia, Italy). The expected range for pepsinogen $\mathrm{I}$ in healthy volunteers is $28-175 \mu g / l$.

\section{Statistical analysis}

The $\chi^{2}$ test or Fisher's exact test was used to compare the rate of subsequent ulcer development with the 13 clinical, serological, histological, and bacterial variables. These variables included the following: age $(<60$ or $\geqslant 60$ years), sex, blood type (O type or non-O type), history of smoking, history of alcohol consumption ( $<80 \mathrm{~g} /$ day or $\geqslant 80 \mathrm{~g} /$ day $)$, ingestion of coffee $(<1 \mathrm{cup} /$ day or $\geqslant 1$ cup/day), ingestion of tea $(<1$ cup/ day or $\geqslant 1$ cup/day), patterns of dyspeptic symptoms (prevalent pain subgroup or other subgroup), severity of dyspepsia (score $<4$ or $\geqslant 4$ ), ingestion of NSAID, hypergastrinaemia (serum gastrin $>89 \mathrm{pg} / \mathrm{ml}$ ), hyperpepsinogenaemia (serum pepsinogen I $>175 \mu \mathrm{g} / \mathrm{l}$ ), and $H$ pylori status (absence or presence). A p value $<0.05$ was considered to be significant. Those variables found to be significant by univariate analysis were subsequently assessed by a stepwise logistic regression method to identify independent risk factors for subsequent development of peptic ulcers in NUD patients.

To investigate differences in clinical outcome between $H$ pylori positive and $H$ pylori negative NUD, rates of ulcer formation, erosion formation, and symptom resolution were compared by $\chi^{2}$ test. Resolution of symptoms ${ }^{13}$ was defined as a score $<3$ at the end of the second month and thereafter.

\section{RESULTS}

From May 1997 to April 1999, we enrolled 395 patients with NUD. Among them, 186 were excluded for the following reasons: past history of peptic ulcer (4l patients), ingestion of NSAID (six patients) or acid reducing agents (four patients) before entry, serious medical condition ( 16 patients), pancreaticobiliary tract disease ( 15 patients), declining to participate ( 18 patients), and being assigned to anti- $H$ pylori therapy ( 86 patients).

Ninety five of the 209 eligible NUD patients were $H$ pylori positive. During the prospective two year follow up period, 16 of the 209 patients (7.7\%) developed peptic ulcers. Among the 16 ulcers, $12(75.0 \%)$ presented with dyspepsia and one $(6.3 \%)$ presented with bleeding. The other three (18.8\%) were asymptomatic.

\section{Risk factors for subsequent development of peptic ulceration}

Univariate analysis of the 13 clinical, serological, and bacterial factors (table 1) demonstrated that the following three were significantly associated with subsequent development of peptic ulceration: advanced age $(\mathrm{p}=0.01)$, H pylori infection $(\mathrm{p}<0.05)$, and ingestion of NSAID $(\mathrm{p}<0.01)$. The other factors ( $\mathrm{sex}$, blood type, history of smoking, alcohol consumption, ingestion of coffee, ingestion of tea, patterns or severity of dyspeptic symptoms, hypergastrinaemia, and hyperpepsinogenaemia) did not increase the risk of ulcer formation (table 1).

Multivariate analysis with stepwise logistic regression showed that all three factors were independently significant in predicting subsequent development of peptic ulcer (table 2). The odds ratios of the three parameters were 2.90 (95\% confidence interval (CI) 0.98-8.50), 3.59 (95\% CI 1.07-12.05), and 4.46 (95\% CI 1.31-15.17), respectively. NUD patients with a 
Table 1 Univariate analysis of risk factors for subsequent development of peptic ulceration in 209 non-ulcer dyspepsia patients

\begin{tabular}{|c|c|c|c|}
\hline Principal parameter & No of patients & $\begin{array}{l}\text { Rate of peptic } \\
\text { ulceration (\%) }\end{array}$ & $\mathrm{p}$ Value \\
\hline \multicolumn{4}{|l|}{ Clinical factors } \\
\hline \multicolumn{4}{|l|}{ Age } \\
\hline$<60 y$ & $\begin{array}{r}150 \\
50\end{array}$ & $\begin{array}{r}4.7 \\
15.2\end{array}$ & 0.01 * \\
\hline$\geqslant 60 \mathrm{yr}$ & 59 & 15.3 & \\
\hline \multicolumn{4}{|l|}{ Sex } \\
\hline Female & 113 & 7.1 & 0.734 \\
\hline Male & 96 & 8.3 & \\
\hline \multicolumn{4}{|l|}{ Blood type } \\
\hline Non-O & 124 & 8.9 & 0.425 \\
\hline 0 & 85 & 5.9 & \\
\hline \multicolumn{4}{|l|}{ Smoking } \\
\hline$(-)$ & 170 & 7.6 & 1.000 \\
\hline \multirow{2}{*}{\multicolumn{4}{|c|}{ Alcohol consumption }} \\
\hline & & & \\
\hline$(-)$ & 191 & 7.9 & 1.000 \\
\hline $1+1$ & 18 & 5.6 & \\
\hline \multicolumn{4}{|l|}{ Coffee consumption } \\
\hline$(-)$ & 177 & 7.3 & 0.717 \\
\hline $1+1$ & 32 & 9.4 & \\
\hline \multicolumn{4}{|l|}{ Ingestion of tea } \\
\hline$(-)$ & 155 & 5.8 & 0.089 \\
\hline $1+1$ & 54 & 13.0 & \\
\hline \multicolumn{4}{|l|}{ Patterns of symptoms } \\
\hline Ulcer-like subgroup & 72 & 6.9 & 0.779 \\
\hline Other subgroups & 137 & 8.0 & \\
\hline \multicolumn{4}{|l|}{ Severity of symptom } \\
\hline Mild & 90 & 8.9 & 0.560 \\
\hline Severe & 119 & 6.7 & \\
\hline \multicolumn{4}{|l|}{ Ingestion of NSAID } \\
\hline$(-)$ & 186 & 5.9 & $0.007 *$ \\
\hline $1+1$ & 23 & 21.7 & \\
\hline \multicolumn{4}{|l|}{ Serological factors } \\
\hline \multicolumn{4}{|l|}{ Hypergastrinaemia } \\
\hline$(-)$ & 181 & 7.7 & 1.000 \\
\hline $1+1$ & 28 & 7.1 & \\
\hline \multicolumn{4}{|l|}{ Hyperpepsinogenaemia } \\
\hline$(-)$ & 203 & 3.0 & 1.000 \\
\hline $1+1$ & 6 & 0.0 & \\
\hline \multirow{2}{*}{\multicolumn{4}{|c|}{ Bacterial factor }} \\
\hline H pylori & & & \\
\hline$(-1)$ & 114 & 3.5 & $0.018 *$ \\
\hline $1+1$ & 95 & 12.6 & \\
\hline
\end{tabular}

Table 2 Stepwise logistic regression of risk factors for subsequent development of peptic ulceration in 209 non-ulcer dyspepsia patients

\begin{tabular}{lllll}
\hline Risk factor & Coefficient & Standard error & Odds ratio $(95 \% \mathrm{Cl})$ & $\mathrm{p}$ Value \\
\hline Advanced age & 1.0630 & 0.5497 & $2.90(0.98-8.50)$ & 0.053 \\
H pylori infection & 1.2772 & 0.6185 & $3.59(1.07-12.05)$ & 0.039 \\
Use of NSAID & 1.4946 & 0.6250 & $4.46(1.31-15.17)$ & 0.017 \\
\hline
\end{tabular}

NSAID, non-steroidal anti-inflammatory drugs; $\mathrm{Cl}$, confidence interval.

history of NSAID use and H pylori infection had a 36.4\% (4/11) ulcer risk while an NSAID user without $H$ pylori infection had an ulcer rate of only $8.3 \%$ (1/12). NUD patients with all three risk factors had a 75\% (3/4) risk of developing peptic ulcers but the ulcer incidence in patients without any of the risk parameters was only $1.2 \%(1 / 84)$.

\section{Comparison of $\boldsymbol{H}$ pylori positive and $\boldsymbol{H}$ pylori negative NUD patients}

$H$ pylori positive and negative NUD patients were comparable at baseline regarding sex, blood type, smoking habits, alcohol, coffee and tea consumption, pattern and severity of symptoms, and serum gastrin levels. However, the $H$ pylori infected subjects were significantly older than the $H$ pylori negative subjects $(52.7$ (15.5) v 45.4 (16.6) years old; $\mathrm{p}<0.01)$. In addition, patients with $H$ pylori positive NUD had higher levels of serum pepsinogen I (77.6 (31.7) $v 66.1(40.7) \mu \mathrm{g} / \mathrm{l} ; \mathrm{p}<0.05)$ compared with patients with $H$ pylori negative NUD.

During the two year follow up period, two of the $114 \mathrm{H}$ pylori negative patients $(1.8 \%)$ became infected with $H$ pylori whereas spontaneous loss of $H$ pylori did not occur in any of the $95 \mathrm{H}$ pylori positive patients. Symptom scores in the $114 \mathrm{H}$ pylori negative group decreased significantly during the follow up period (all $\mathrm{p}<0.00 \mathrm{l}$ compared with initial symptom score). Meanwhile, the symptom score in $114 H$ pylori positive patients also significantly decreased during follow up (all $\mathrm{p}<0.001$ compared with the initial score). 
Table 3 Long term outcome of Helicobacter pylori positive and $H$ pylori negative non-ulcer dyspepsia (NUD) patients

\begin{tabular}{lllll}
\hline & $\begin{array}{l}\text { H pylori(+) NUD } \\
(\mathrm{n}=95)\end{array}$ & $\begin{array}{l}\text { H pylori(-) NUD } \\
(\mathrm{n}=114)\end{array}$ & $\begin{array}{l}\text { Difference } \\
(95 \% \mathrm{Cl})\end{array}$ & $\mathrm{p}$ Value \\
\hline Resolution of symptoms & $55 / 95(57.9 \%)$ & $56 / 114(49.1 \%)$ & $8 \%(-5$ to 22$)$ & 0.206 \\
Erosion & $22 / 95(23.2 \%)$ & $14 / 114(12.3 \%)$ & $11 \%(1-22)$ & 0.038 \\
Ulcer & $12 / 95(12.6 \%)$ & $4 / 114(3.5 \%)$ & $9 \%(1-16)$ & 0.018 \\
$\quad$ Oesophagus & 4 & 0 & & \\
$\quad \begin{array}{l}\text { Stomach } \\
\text { Fundus }\end{array}$ & 1 & 0 & & \\
$\quad$ Body & 1 & 0 & & \\
$\quad$ Angle & 0 & 0 & & \\
$\quad$ Antrum & 5 & 3 & & \\
Duodenum & & & & \\
$\quad$ Bulb & 1 & 1 & & \\
$\quad$ 2nd portion & 0 & 0 & & \\
\hline
\end{tabular}

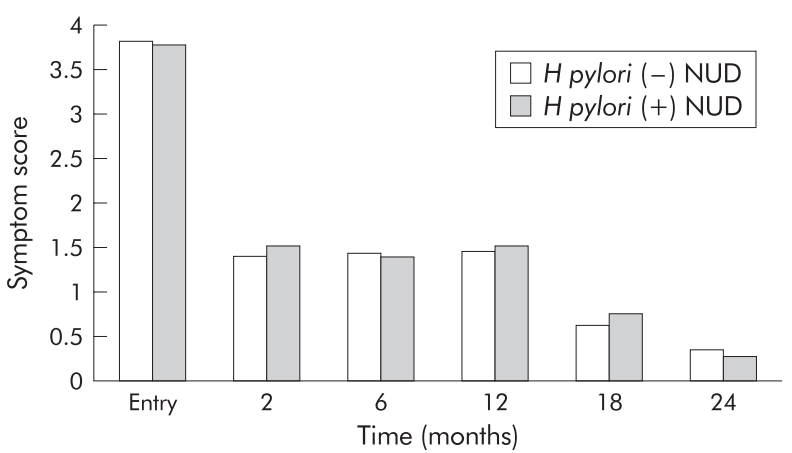

Figure 1 There were no significant differences in symptoms scores at each follow up time point between Helicobacter pylori positive and negative non-ulcer dyspepsia (NUD) patients (all $p$ values $>0.05$ ).

The resolution rate of symptoms in the $H$ pylori negative NUD patients was similar to that in the $H$ pylori positive patients ( $49.1 \% \vee 57.9 \% ; \mathrm{p}>0.05)$, and the two groups of NUD patients did not significantly differ in symptom scores at each follow up time point (table 3, fig 1). However, the rates of subsequent peptic ulcer (mucosal break $\geqslant 5 \mathrm{~mm}$ ) and erosion (mucosal break $<5 \mathrm{~mm}$ ) development were significantly higher in the $H$ pylori positive patients than in the $H$ pylori negative patients (ulcer $12.6 \%$ v 3.5\%, 95\% CI $1-16$; erosion $23.2 \%$ v $12.3 \%, 95 \%$ CI $1-21)$.

\section{DISCUSSION}

Several studies ${ }^{4-7}{ }^{10}$ have shown that a significant proportion of NUD patients develop peptic ulcers after long term follow up. Most of the studies which have investigated the relationship between peptic ulcer and NUD however were based on re-examination of symptomatic patients only. It is therefore possible that some silent ulcers escaped detection in these studies. $^{47_{10}}$ In a retrospective study by Lindell and colleagues, ${ }^{7}$ the cumulative 10 year risk of symptomatic peptic ulcer was only $2 \%$. In contrast, Matysiak-Budnik and colleagues ${ }^{6}$ reported that the five year cumulative probability of peptic ulcer was as high as $21 \%$. The wide variance of ulcer risk makes it extremely difficult for physicians to decide whether repetitive endoscopy is necessary in NUD patients with recurrent symptoms. Our current study prospectively followed 209 NUD patients for two years, and discovered that the cumulative risk of peptic ulcer development in NUD patients was $7.7 \%(16 / 209)$. It is important to point out that the present study was based on two routine follow up endoscopies and on re-examination of symptomatic patients. Among 16 ulcers detected in this study, three (18.8\%) were asymptomatic. This observation strongly suggests that most previous studies failed to detect silent peptic ulcers. One might question whether the presence of an ulcer crater without appreciable discomfort and that does not interfere with activities can be considered a disease entity. In fact, ulcer patients may have life threatening complications, such as bleeding or perforation, without obvious preceding symptoms. Asymptomatic ulcers should therefore not be ignored in a clinical follow up study.

Although it is well known that some NUD patients may develop peptic ulcers during long term follow up, no published work has simultaneously evaluated clinical, serological, and bacterial factors by multivariate analysis, and quantitatively assessed the risk factors for subsequent ulcer formation. In this study, univariate analysis showed that $H$ pylori infection, NSAID use, and advanced age were associated with ulcer formation, and multivariate analysis demonstrated that they were independent risk factors for ulcer development. When considering the three factors according to their odds ratios, use of NSAID was the strongest factor in predicting ulcer formation. It should be emphasised however that a combination of risk factors may provide a more reliable prediction of ulcer formation than a single factor. We found that NUD patients with a history of NSAID use and H pylori infection had a $36.4 \%$ (4/11) ulcer risk while an NSAID user without $H$ pylori infection had an ulcer rate of only $8.3 \%$ (1/12). Interestingly, older NUD patients taking NSAID and with $H$ pylori infection had a $75 \%(3 / 4)$ risk of developing peptic ulcers in the two year follow up period but younger patients without $H$ pylori infection and not using NSAID had an ulcer rate of only $1.2 \%(1 / 82)$. These novel findings imply that repeat endoscopy is rarely advantageous when symptoms recur in an NUD patient lacking any of the aforementioned three risk factors. In contrast, follow up endoscopy is strongly indicated in an NUD patient with multiple risk factors for ulcer formation.

The prevalence of H pylori infection in NUD ranges from 35\% to $87 \% .{ }^{90}$ Currently, there is still no consensus on whether eradication of $H$ pylori relieves dyspeptic symptoms in NUD patients. ${ }^{9-11}$ It is important to note that approximately half of NUD patients are not infected by $H$ pylori, and their clinical courses have never been investigated yet. Another major aim of this pilot study is therefore to establish the natural history of $H$ pylori negative NUD patients. During the prospective two year follow up period, relapse of dyspeptic symptoms occurred in 56 of $114(49.1 \%) H$ pylori negative patients. Four of these NUD patients $(3.5 \%)$ developed peptic ulcers (three gastric and one duodenal). As in our previous report ${ }^{21}$ and several other studies, ${ }^{22}{ }^{23} \mathrm{H}$ pylori negative NUD patients were younger than $H$ pylori positive patients. Serum pepsinogen I levels were significantly lower in $H$ pylori negative NUD patients than in $H$ pylori positive patients. ${ }^{21}$ In the two year follow up period, the 
resolution rate of symptoms in the $H$ pylori negative NUD patients was similar to that in the $H$ pylori positive patients (49.1\% $v 57.9 \% ; \mathrm{p}>0.05)$, and the two groups of NUD patients did not significantly differ in symptom scores at each follow up time point. However, it is important to note that both the rates of subsequent peptic ulcer and erosion development were significantly higher in $H$ pylori positive patients than in $H$ pylori negative patients (ulcer $12.6 \%$ v 3.5\%, 95\% CI 1-16; erosion $23.2 \% v 12.3 \%, 95 \%$ CI $1-21$ ). Our study is the first long term cohort study confirming that $H$ pylori status influences the risk of ulcers in NUD patients. The results are consistent with the report of Sipponen and colleagues, ${ }^{4}$ in which the 10 year cumulative probability of peptic ulcer in patients with histological gastritis was significantly higher than that in patients with normal gastric mucosa. However, the exact $H$ pylori status was not examined in their study. Recently, a well conducted study by McColl and colleagues ${ }^{10}$ also demonstrated that four of 308 patients with $H$ pylori related NUD developed peptic ulcer during the one year follow up period. All patients developing peptic ulceration at repeat endoscopy were initially assigned to the non-eradication group but not the $H$ pylori eradication group. The aforementioned observation and our study suggest that it merits further investigation to clarify whether eradicating H pylori can prevent subsequent ulcer development in aged NUD patients.

Multiple factors are possibly involved in the development of gastroduodenal ulcerations. Although $H$ pylori infection and use of NSAID are well known critical factors in the pathogenesis, other clinical elements (for example, smoking and genetic factors ${ }^{24}$ ) may contribute to the development of ulcer diseases. Additionally, an increased serum level of pepsinogen was reported as a highly significant risk factor for duodenal ulcer development. ${ }^{25}$ In the current study, we disclosed that advanced age increased the ulcer risk in patients with NUD but other factors were not independent risk factors for subsequent ulcer development. Several other independent studies ${ }^{26}{ }^{27}$ have also highlighted the importance of age in ulcer formation. Garcia-Rodriguez and Jick ${ }^{26}$ proved that advanced age increased the risk of ulcer complications in NSAID users. Simons ${ }^{27}$ demonstrated that advanced age was an independent risk factor for stress ulceration after trauma. The reason why the aged stomach is more vulnerable to injury remains unclear. It likely that in the older age group, more patients had occult NSAID intake. However, the increased ulcer risk in the elderly is also probably due to age related changes in the gastric mucosal defence. ${ }^{28}$ Two studies in humans ${ }^{29}{ }^{30}$ have demonstrated that gastric mucosal prostaglandin content declines with age. Feldman and Cryer $^{30}$ have also disclosed that advanced age is associated with a significant decline in gastric bicarbonate, sodium ions, and non-parietal fluid secretion. Thus aging is associated with selective as well as specific changes in the gastric mucosal defences that may predispose aged NUD patients to develop peptic ulceration. It is therefore important to adopt some strategies (for example, concurrent administration of proton pump inhibitors, use of the cyclo-oxygenase 2 specific inhibitors) to prevent ulcer development in aged NUD patients who need chronic NSAID therapy.

In conclusion, a small but significant proportion of NUD patients developed peptic ulcer after long term follow up. Advanced age, $H$ pylori infection, and use of NSAID were independent risk factors for subsequent ulcer formation. Follow up endoscopy is strongly indicated in an NUD patient with multiple risk factors for ulcer formation.

\section{ACKNOWLEDGEMENTS}

The authors express their deep appreciation to Drs Chi-Pin Lin, JengJie Pzeng, I-Fei Huang, Zough-Sham Wang, Ying-Tsun Lin, and Miss Chia-Li Yu for their assistance in clinical follow up of the patients, and to Professor Luo-Ping Ger for help with statistics. The study was supported by research grant Nos NSC-89-2314-B-075B-008 from the
National Science Council, Taiwan, and VGHKS-90-4l from the Research Foundation of Kaohsiung Veterans General Hospital.

\section{Authors' affiliations}

P-I Hsu, K-H Lai, G-H Lo, C-C Lo, H-C Chen, W-L Tsai, Division of Gastroenterology, Kaohsiung Veterans General Hospital, Taiwan, ROC H-H Tseng, H-S Jou, Department of Internal Medicine, Department of Pathology, Kaohsiung Veterans General Hospital, Taiwan, ROC

N-J Peng, Department of Nuclear Medicine, Kaohsiung Veterans General Hospital, Taiwan, ROC

C-H Chien, Graduate Institute of Physiology, National Yang-Ming University, Taiwan, ROC

J-L Chen, Department of the Health Care and Hospital Administration, Chia-Nan University of Pharmacy and Science, Taiwan, ROC

P-N Hsu, Graduate Institute of Immunology, National Taiwan University, Taiwan, ROC

\section{REFERENCES}

1 Talley NJ, Zinsmeister AR, Schleck CD, et al. Dyspepsia and dyspepsia subgroups: a population-based study. Gastroenterology 1992;102: 1259-68.

2 Dorssman DA, Li Z, Andruzzi E, et al. US householder survey of functional gastrointestinal disorders: Prevalence, sociodemography, and healthy impact. Dig Dis Sci 1993;38:1569-80.

3 Hirakawa K, Adachi K, Amano K, et al. Helicobacter pylori, peptic ulcer and non-ulcer dyspepsia. J Gastroenterol Hepatol 1999; 14:1083-7.

4 Sipponen $\mathbf{P}$, Varis $\mathrm{K}$, Fraki $\mathrm{O}$, et al. Cumulative 10-year risk of symptomatic duodenal and gastric ulcer in patients with or without chronic gastritis. Scand J Gastroenterol 1990;25:966-73.

5 Gilvarry J, Buckley MM, Beattie S, et al. Eradication of Helicobacter pylori affects symptoms in non-ulcer dyspepsia. Scand J Gastroenterol 1997; 32:535-40.

6 Matysiak-Budnik T, Poneiwierka E, Gasciniak G, et al. A 5-year follow-up study of chronic gastritis patients. Ir J Med Sci 1992;161/suppl 10):59.

7 Lindell GH, Celebioglu F, Graffner HO. Non-ulcer dyspepsia in the long-term perspective. Eur J Gastroenterol Hepatol 1995;7:829-33.

8 Perura DA. The report of the Digestive Health Initiative ${ }^{S M}$ International Update Conference on Helicobacter pylori. Gastroenterology 1997;113:S4-8

9 Armstrong D. Helicobacter pylori infection and dyspepsia. Scand J Gastroenterol 1996;31(suppl 215):38-47.

10 McColl K, Murray L, El-Omar E, et al. Symptomatic benefit from eradication of Helicobacter pylori infection in patients with nonulcer dyspepsia. N Engl J Med 1998;339:1869-74.

11 Blum AL, Talley NJ, O'Morain C, et al. Lack of effect of treating Helicobacter pylori infection in patients with nonulcer dyspepsia. N Engl J Med 1998;339:1875-81

12 Manning AP, Thompson WG, Heaton KW, et al. Toward positive diagnosis of the irritable bowel. BM 1978;2:653-4.

13 Hsu PI, Lai KH, Tseng HH, et al. Eradication of Helicobacter pylori prevents ulcer development in patients with ulcer-like functional dyspepsia. Aliment Pharmacol Ther 2001;15:195-201.

14 Hsu PI, Lai KH, Tseng HH, et al. Correlation of serum immunoglobulin G Helicobacter pylori antibody titers with histologic and endoscopic findings in patients with dyspepsia. J Clin Gastroenterol 1997;25:587-91.

15 Hsu PI, Lai KH, Tseng HH, et al. Risk factors for presentation with bleeding in patients with Helciobacter pylori-related peptic ulcer diseases. J Clin Gastroenterol 2000;30:386-91.

16 Dixon MF, Genta RM, Yardly JH, et al. Classification and grading of gastritis. The Updated Sydney System. Am J Surg Pathol 1996;20:1161-81

17 Dominguez-Munoz JE, Leodolter A, Sauerbruch T, et al. A citric acid solution is an optimal test drink in the 13C-urea breath test for the diagnosis of Helicobacter pylori infection. Gut 1997;40:459-62.

18 Tucci A, Corinaldesi R, Stanghellini V, et al. Helicobacter pylori infection and gastric function in patients with chronic idiopathic dyspepsia. Gastroenterology 1992;103:768-74.

19 Tosetti C, Stanhellini V, Paternico A, et al. Overlapping irritable bowel syndrome and delayed gastric emptying identify different subgroups among patients with functional dyspepsia. Gut 1996;39(suppl 3):A219.

20 Lambert JR. The role of Helicobacter pylori in nonulcer dyspepsia. Gastroenterol Clin North Am 1993;22:151.

21 Lo CC, Hsu PI, Lo GH, et al. Comparison of clinical, serological and histological findings between non-ulcer dyspepsia patients with and without Helicobacter pylori infection. J Gastroenterol Hepatol $2001 ; 16: 276-81$

22 Blum AL, Arnold R, Stolte M, et al. Short course acid suppressive treatment for patients with functional dyspepsia: results depend on Helicobacter pylori status. Gut 2000;47:473-80.

23 Bennet EJ, Plesse C, Palmer K, et al. Functional gastrointestinal disorders: psychological, social, and somatic features. Gut 1998;42:414-20.

24 Go MF. What are the host factors that place an individual at risk for Helicobacter pylori-associated disease? Gastroenterology 1997;113:S15-20. 
25 Samloff IM. Peptic ulcer: the many proteinases of aggression. Gastroenterology 1989;96:586-95.

26 Garcia-Rodriguez LA, Jick H. Risk of upper gastrointestinal bleeding and perforation associated with individual non-steroidal anti-inflammatory drugs. Lancet 1994;343:769-72.

27 Simons RK. A risk analysis of stress ulcerations after trauma. J Trauma 1995;39:289-93.
28 Lee $M$, Feldman M. The aging stomach: implications for NSAID gastropathy. Gut 1997;4:425-6.

29 Cryer B, Redfern JS, Goldschmeidt M, et al. Effect of aging on gastric and duodenal mucosal prostaglandin concentrations in humans. Gastroenterology 1992;102:1118-23.

30 Feldman $M$, Cryer B. Effects of normal aging on gastric nonparietal fluid and electrolyte secretion in humans. Gerontology 1998;44:222-7.

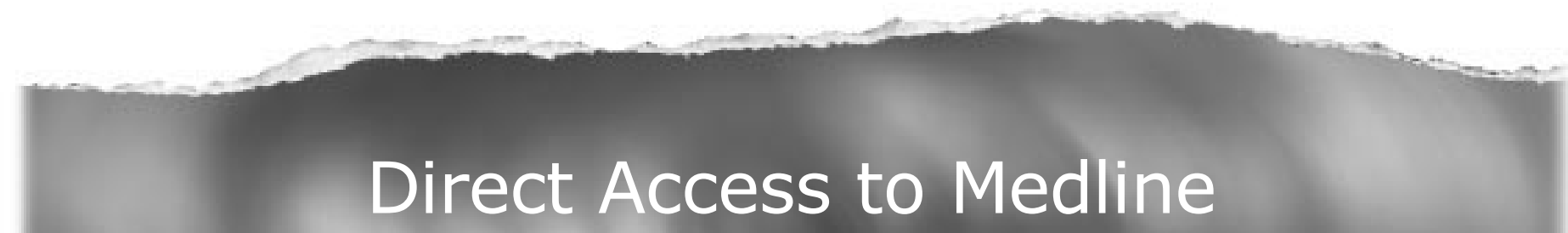

\section{Medline}

Link to Medline from the homepage and get straight into the National Library of Medicine's premier bibliographic database. Medline allows you to search across 9 million records of bibliographic citations and author abstracts from approximately 3,900 current biomedical journals.

www.gutjnl.com 